This document is confidential and is proprietary to the American Chemical Society and its authors. Do not copy or disclose without written permission. If you have received this item in error, notify the sender and delete all copies.

\title{
Supporting Information for Effect of Water Content on Sodium Chloride Sorption in Cross-Linked Cation Exchange Membranes
}

\begin{tabular}{|r|l|}
\hline Journal: & Macromolecules \\
\hline Manuscript ID & ma-2019-00737p.R1 \\
\hline Manuscript Type: & Additions and Corrections \\
\hline Date Submitted by the & 17-Apr-2019 \\
\hline Complete List of Authors: & $\begin{array}{l}\text { Jang, Euisoung; University of Texas at Austin, } \\
\text { Kamcev, Jovan; University of Texas at Austin } \\
\text { Kobayashi, Kentaro; University of Texas at Austin } \\
\text { Yan, Ni; University of Texas at Austin } \\
\text { Sujanani, Rahul; University of Texas at Austin } \\
\text { Talley, Samantha; Virginia Polytechnic Institute and State University, } \\
\text { Chemistry } \\
\text { Moore, Robert; Virginia Polytechnic Institute and State University, } \\
\text { Department of Chemistry } \\
\text { Paul, Donald; University of Texas at Austin, Chemical Engineering } \\
\text { Department and Texas Material Institute; I\&EC Research, Dept. of } \\
\text { Chemical Engineering } \\
\text { Freeman, Benny; University of Texas at Austin, McKetta Department of } \\
\text { Chemical Engineering; }\end{array}$ \\
\hline
\end{tabular}

\section{SCHOLARONE Manuscripts}




\title{
Supporting Information
}

\section{Effect of Water Content on Sodium Chloride Sorption in Cross-Linked Cation Exchange Membranes}

\author{
Eui-Soung Jang, ${ }^{\dagger}$ Jovan Kamcev,${ }^{\dagger}$ Kentaro Kobayashi, ${ }^{\dagger}$ Ni Yan, ${ }^{\dagger}$ \\ Rahul Sujanani, ${ }^{\dagger}$ Samantha J. Talley, $₫$ Robert B. Moore, \\ Donald R. Paul ${ }^{\dagger}$, and Benny D. Freeman*† \\ †Department of Chemical Engineering, Texas Materials Institute, Center for Energy and \\ Environmental Resources, and Center for Research in Water Resources \\ The University of Texas at Austin \\ 10100 Burnet Road, Bldg. 133 - CEER \\ Austin, TX 78758 USA \\ \#Department of Chemistry, Macromolecules Innovation Institute \\ Virginia Tech \\ Blacksburg, VA 24061 USA
}

*To whom correspondence should be addressed: freeman@che.utexas.edu

(Tel: +1-512-232-2802, Fax: +1-512-232-2807) 


\section{S1. Water uptake and water volume fraction in the CEMs equilibrated with $\mathrm{NaCl}$ solutions}

Table S1 shows equilibrium water uptake values for the CEMs as a function of external

$\mathrm{NaCl}$ concentration. The water volume fraction in the membrane, $\phi_{w}$, was calculated based on volume additivity as follows: ${ }^{1}$

$$
\phi_{w}=\frac{w_{u}}{w_{u}+\rho_{w} / \rho_{p}}
$$

where $w_{u}$ is the mass water uptake (i.e., g water/g dry polymer), $\rho_{P}$ is the dry polymer density, and $\rho_{W}$ is the water density (i.e., $1.0 \mathrm{~g} / \mathrm{cm}^{3}$ ). The dry polymer densities and water uptake of these polymers are presented in Table 1 in the main text. 
Table S1. Water content in the CEMs.

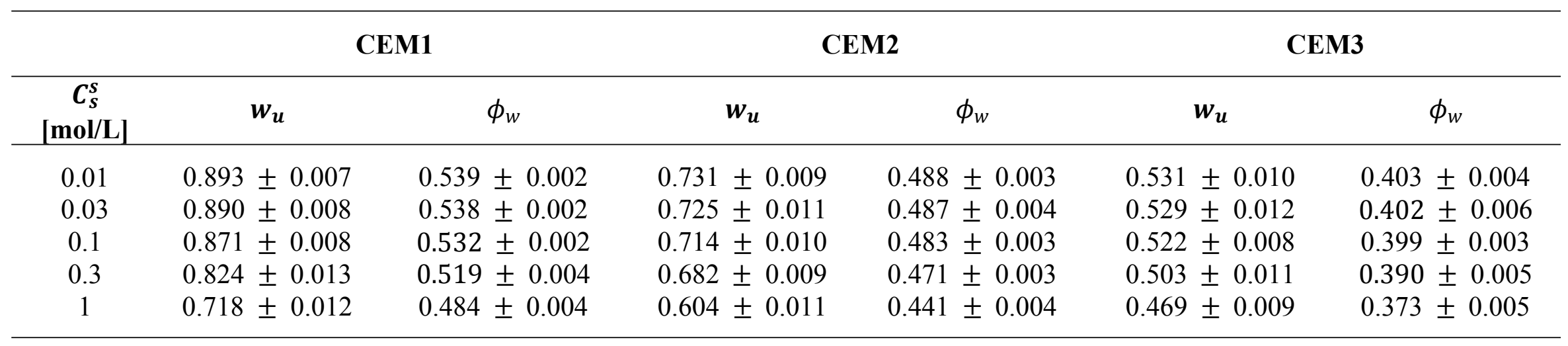




\section{S2. Cation and anion concentrations in the samples}

Equilibrium molar concentrations of counter-ions (i.e., $\mathrm{Na}^{+}$ion) and co-ions (i.e., $\mathrm{Cl}^{-}$ion) as a function of external salt concentration are recorded in Tables S2 and S3, respectively. External salt solution concentration, $C_{s}^{S}$, is expressed as moles of salt per liter of solution. Ion concentrations in the membrane are reported as moles of ions per liter of swollen polymer (i.e., polymer + water + ions $), C_{i}^{m, p}$, and as moles of ions per liter of sorbed water in swollen polymer (i.e., water + ions), $C_{i}^{m, w}$. 
Table S2. Membrane counter-ion concentration as a function of external $\mathrm{NaCl}$ concentration.

\begin{tabular}{|c|c|c|c|c|c|c|}
\hline \multirow[b]{2}{*}{$\begin{array}{c}C_{s}^{S} \\
{[\mathrm{~mol} / \mathrm{L}]}\end{array}$} & \multicolumn{2}{|c|}{ CEM1 } & \multicolumn{2}{|c|}{ CEM2 } & \multicolumn{2}{|c|}{ CEM3 } \\
\hline & $C_{g}^{m, p}$ & $C_{g}^{m, w}$ & $C_{g}^{m, p}$ & $C_{g}^{m, w}$ & $C_{g}^{m, p}$ & $C_{g}^{m, w}$ \\
\hline 0.01 & $0.90 \pm 0.01$ & $1.67 \pm 0.01$ & $0.83 \pm 0.02$ & $1.70 \pm 0.03$ & $0.72 \pm 0.01$ & $1.74 \pm 0.04$ \\
\hline 0.03 & $0.91 \pm 0.01$ & $1.70 \pm 0.03$ & $0.83 \pm 0.01$ & $1.71 \pm 0.02$ & $0.72 \pm 0.01$ & $1.75 \pm 0.04$ \\
\hline 0.1 & $0.95 \pm 0.01$ & $1.79 \pm 0.02$ & $0.88 \pm 0.01$ & $1.82 \pm 0.02$ & $0.72 \pm 0.01$ & $1.78 \pm 0.03$ \\
\hline 0.3 & $0.98 \pm 0.01$ & $1.89 \pm 0.02$ & $0.90 \pm 0.01$ & $1.91 \pm 0.03$ & $0.77 \pm 0.01$ & $1.94 \pm 0.04$ \\
\hline 1 & $1.16 \pm 0.02$ & $2.40 \pm 0.05$ & $1.06 \pm 0.01$ & $2.40 \pm 0.03$ & $0.85 \pm 0.01$ & $2.24 \pm 0.04$ \\
\hline
\end{tabular}

Table S3. Membrane co-ion concentration as a function of external $\mathrm{NaCl}$ concentration.

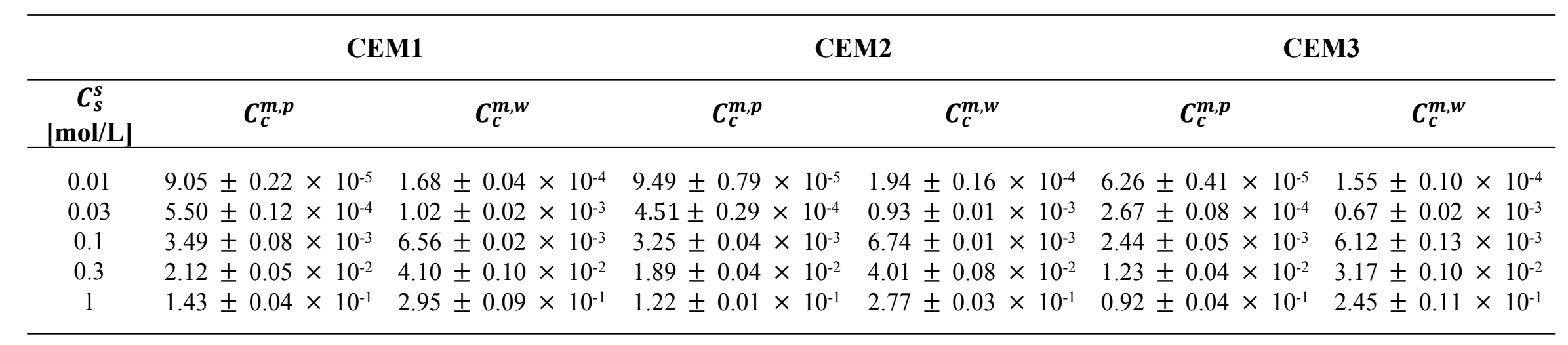




\section{S3. Quantitative analysis of contributions from co-ion and osmotic desweling to increase in counter-ion concentration}

The counter-ion concentration increases as external salt solution concentration increases due to increasing co-ion concentration and osmotic deswelling. The membrane co-ion concentration at $0.01 \mathrm{M}$ external $\mathrm{NaCl}$ concentration is effectively negligible compared to counter-ion concentration. Thus, the co-ion and counter-ion concentrations at $0.01 \mathrm{M}$ external $\mathrm{NaCl}$ concentration, $C_{-, 0.01}^{m, w}$ and $C_{+, 0.01}^{m, w}$, respectively, were used as a baseline to calculate the contribution from co-ion concentration to an increase in counter-ion concentration as follows:

$$
\frac{C_{-, x}^{m, w}-C_{-, 0.01}^{m, w}}{C_{+, x}^{m, w}-C_{+, 0.01}^{m, w}} \times 100 \%=\% \text { contribution from co-ion at } x \mathrm{M} \text { external } \mathrm{NaCl} \text { conc }
$$

where $C_{-, x}^{m, w}$ and $C_{+, x}^{m, w}$ are the co-ion and counter-ion concentrations at $x \mathrm{M}$ external $\mathrm{NaCl}$ concentration, respectively. The contribution from osmotic deswelling to the increase in counterion concentration was calculated by deducting the percent contribution from co-ion from $100 \%$.

Table S4. The ratios of contributions from co-ion concentration and osmotic desweling to increase in counter-ion concentration.

\begin{tabular}{ccccccc}
\hline \multirow{2}{*}{$\begin{array}{c}\boldsymbol{s} \boldsymbol{s} \\
{[\mathbf{m o l} / \mathbf{L}]}\end{array}$} & Co-ion & $\begin{array}{c}\text { Osmotic } \\
\text { Deswelling }\end{array}$ & Co-ion & $\begin{array}{c}\text { Osmotic } \\
\text { Deswelling }\end{array}$ & Co-ion & $\begin{array}{c}\text { Osmotic } \\
\text { Deswelling }\end{array}$ \\
\cline { 2 - 6 } & & & & & & \\
$\mathbf{0 . 0 1}$ & - & - & - & - & - & - \\
$\mathbf{0 . 0 3}$ & $3.5 \%$ & $96.5 \%$ & $5.4 \%$ & $94.6 \%$ & $6.1 \%$ & $93.9 \%$ \\
$\mathbf{0 . 1}$ & $5.5 \%$ & $94.5 \%$ & $5.2 \%$ & $94.8 \%$ & $18.0 \%$ & $82.0 \%$ \\
$\mathbf{0 . 3}$ & $18.9 \%$ & $81.1 \%$ & $18.4 \%$ & $81.6 \%$ & $16.4 \%$ & $83.6 \%$ \\
$\mathbf{1}$ & $40.5 \%$ & $59.5 \%$ & $39.3 \%$ & $60.7 \%$ & $49.6 \%$ & $50.4 \%$ \\
& & & & & & \\
\hline
\end{tabular}




\section{S4. Co-ion concentration in the membrane predicted by Manning/Donnan model for homogeneous morphology}

Co-ion concentrations in the membrane predicted by the model for homogeneous morphology and determined by experiment for the CEMs are presented as a function of external $\mathrm{NaCl}$ concentration in Figure $\mathrm{S} 1$. For all three membranes, agreement between the modeling results and experimental values was poor. These results could be due to an incorrect value of the Manning parameter, $\xi$, which is a function of the dielectric constant of the polymer/water mixture, $\varepsilon$, and the distance between fixed charges, $b$ (cf. eq 15). Due to high value of the dielectric constant of water (i.e., 78), the choice of dry polymer dielectric constant does not significantly affect the $\xi$ value as demonstrated in the next section. ${ }^{2,3}$ The distance between fixed charges, $b$, however, could vary significantly depending on polymer morphology. Therefore, the homogeneous polymer morphology assumed to estimate the initial $\xi$ values may not be valid. ${ }^{4}$ 

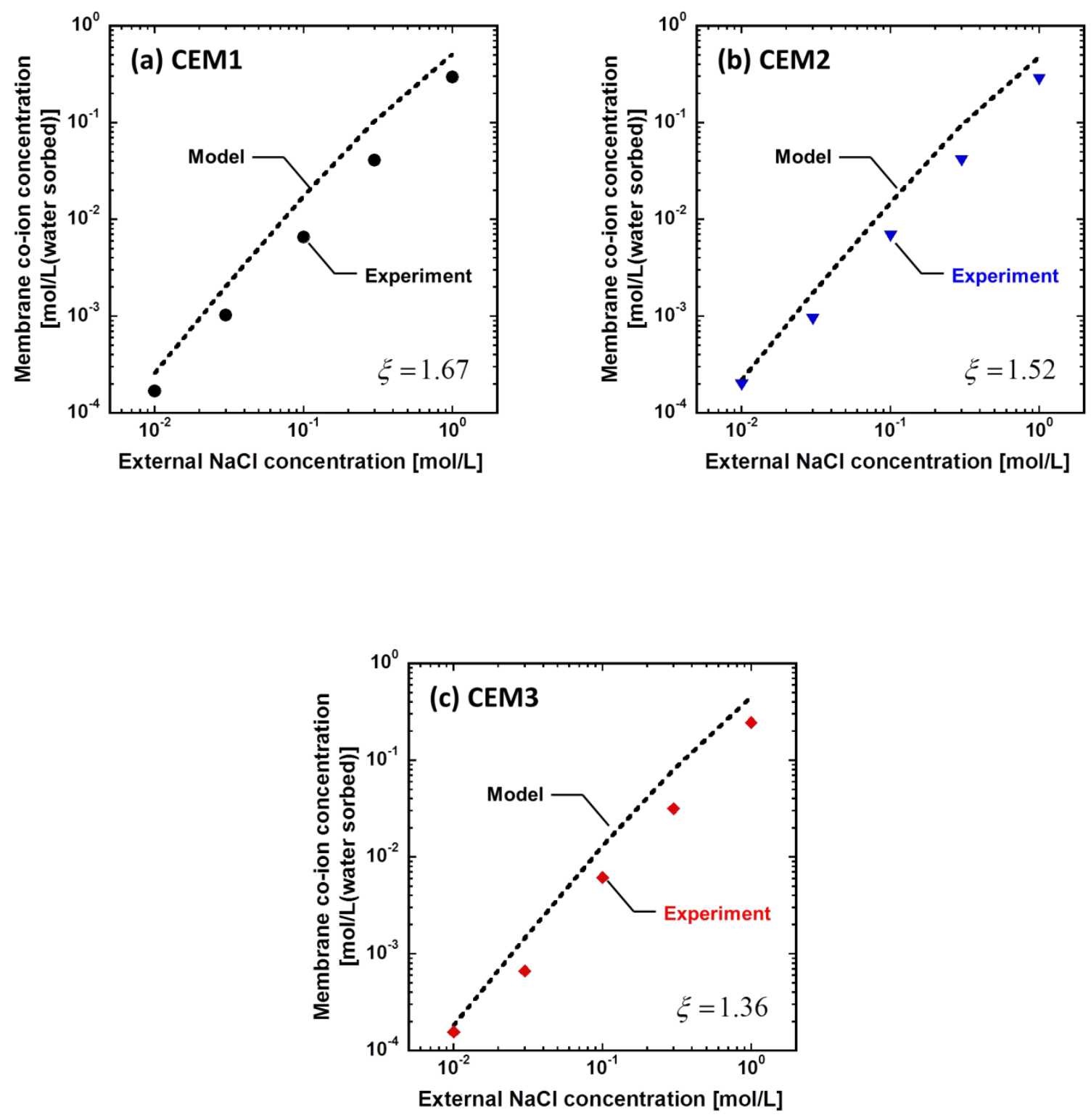

Figure S1. Membrane co-ion concentration as a function of external $\mathrm{NaCl}$ concentration for: (a) CEM1, (b) CEM2, and (c) CEM3. The filled circles represent experimental values and the dashed lines represent values predicted by the Manning/Donnan theory as shown in eq 15 . The Manning parameter, $\xi$, was calculated based on assuming homogeneous morphology. 


\section{S5. Influence of polymer dielectric constant on Manning's parameter}

The dry polymer dielectric constants for the materials considered in this study were chosen based on the values reported in the literature for charged polymers., ${ }^{2,5}$ These values are in the range of 2 to 10 , and a median value (i.e., 6) was used as an estimate of the dry polymer dielectric constant to estimate the dielectric constant of the polymer/water mixture based on a volume fraction weighted average of the dielectric constants of the dry polymer and bulk water. ${ }^{2}$ Since the model using $\xi$ values estimated based on homogeneous materials did not accurately predict co-ion concentration in the membranes, possible $\xi$ value variations induced by choosing different values of the dry polymer dielectric constant were considered. The Manning parameter, $\xi$, as a function of dry polymer dielectric constant for the CEMs is shown in Figure S2. By varying the dry polymer dielectric constant for CEM1 from 2 to 10 , for example, $\xi$ values changes from 1.74 to 1.61 . The magnitude of variation for CEM2 and CEM3 by choosing different dry polymer dielectric constants is similar to that of CEM1. This result is mainly because of high value of water dielectric constant (i.e., 78) and the high water content of these samples, so the choice of dry polymer dielectric constant does not significantly affect $\xi$ values. 


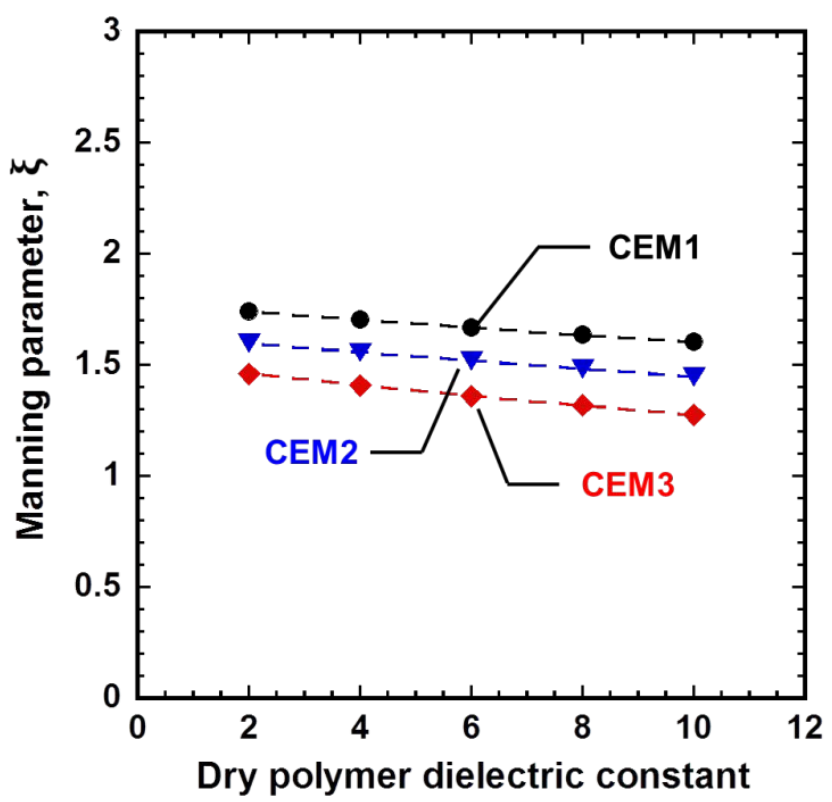

Figure S2. The Manning parameter, $\xi$, as a function of dry polymer dielectric constant for the CEMs. 


\section{S6. UV-vis data for the CEMs}

As discussed in Section S4, predicting co-ion concentration in the CEMs using $\xi$ values estimated based on homogeneous materials did not agree with co-ion concentration determined experimentally. Since the choice of dielectric constant does not significantly affect modeling results as shown in Section S5, these results may be attributed to structural inhomogeneities in the CEMs. Generally, optical turbidity in copolymers is considered as evidence of phase separation. ${ }^{6}$ Although the CEMs were transparent to the naked eye, their transparency was more rigorously tested by UV-vis. Figure S3 (a) shows the UV-vis transmittance of the CEMs in the dried state. To maintain a flat membrane surface, dried sample UV-vis was measured on a quartz plate, which raised the baseline and resulted in transmittance values being somewhat greater than $100 \%$. The data reported here was not corrected for this baseline shift. Homopolymer prepared from the crosslinker (i.e., DEGDMA) was tested as a reference material. The transmittance of all materials did not display any peak in the range of visible light (i.e., $400 \sim 700 \mathrm{~nm}$ ), consistent with these materials being transparent at optical wavelengths. The hydrated CEMs exhibit the same optical characteristics in the visible light range as shown in Figure S3 (b). PDEGDMA was not measured for UV-vis in the hydrated state due to the hydrophobicity of the material and its shrinkage in DI water. 

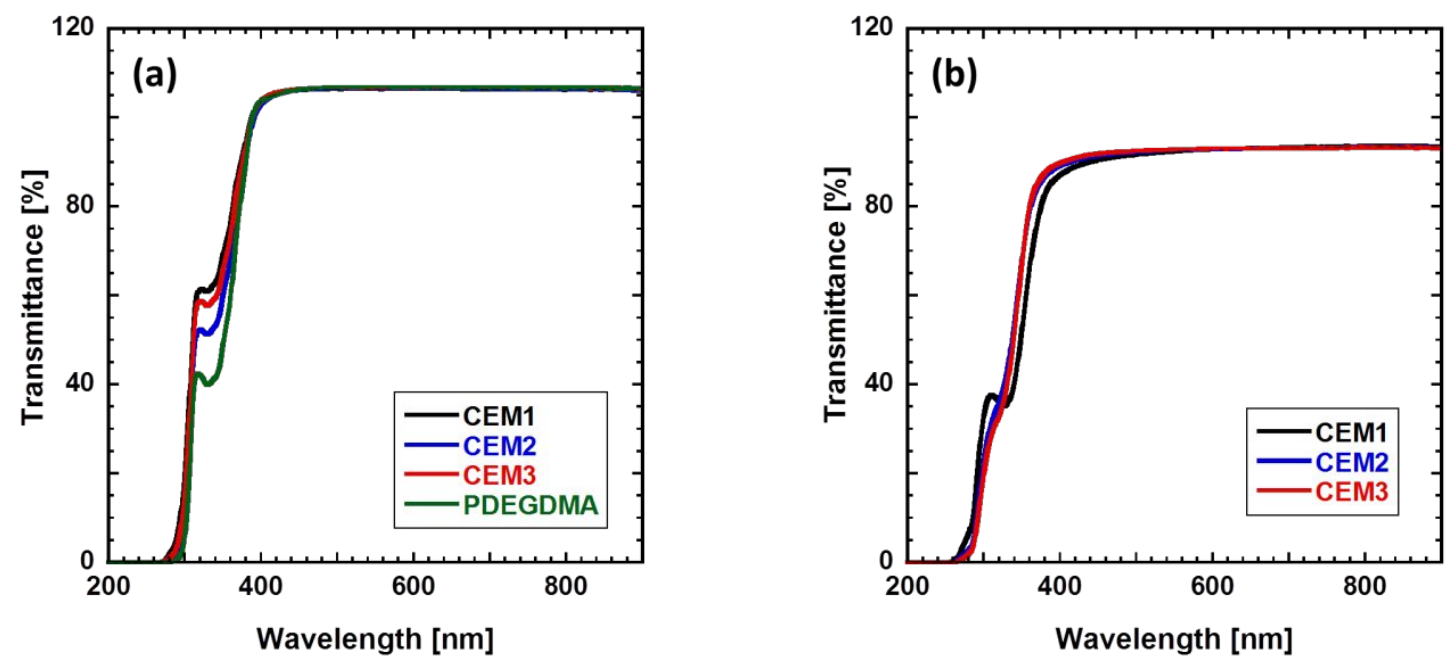

Figure S3. UV-vis transmittance of a series of CEMs: (a) dry and (b) equilibrated with DI water. Dried samples were measured on quartz plates to maintain flat membrane surfaces, which raised the baseline to give transmittance values somewhat above $100 \%$ in (a). PDEGDMA is a homopolymer of DEGDMA (i.e., the cross-linker used for CEMs). 


\section{S7. Fitting to membrane ion activity coefficient data}

$\xi$ values can be fit to experimentally determined ion activity coefficients in the membrane, $\gamma_{+}^{m} \gamma_{-}^{m}$, as a function of $C_{A}^{m, w} / C_{c}^{m, w}$ to find the best fit values for $\xi$. The Manning model expression for ion activity coefficients in a membrane for the case of $\xi<1$ is as follows: ${ }^{7}$

$$
\gamma_{+}^{m} \gamma_{-}^{m}=\exp \left[-\frac{\xi C_{A}^{m, w}}{\left(C_{A}^{m, w}+2 C_{C}^{m, w}\right)}\right]
$$

Best fit values of $\xi$ for CEM1, CEM2, and CEM3 were 0.71, 0.65, and 0.45, respectively, determined using the Curve Fit function in Kaleida Graph software. The results of the fit of this model to the experimentally determined membrane activity coefficients are given in Figure S4. These values were used to calculate co-ion concentration in the membranes reported in Figure 9 in the main manuscript. Furthermore, $\xi$ values fit at each external $\mathrm{NaCl}$ concentration, $C_{s}^{S}$, and corresponding $C_{A}^{m, w} / C_{C}^{m, w}$ are given in Table in Table S5 for comparison. 

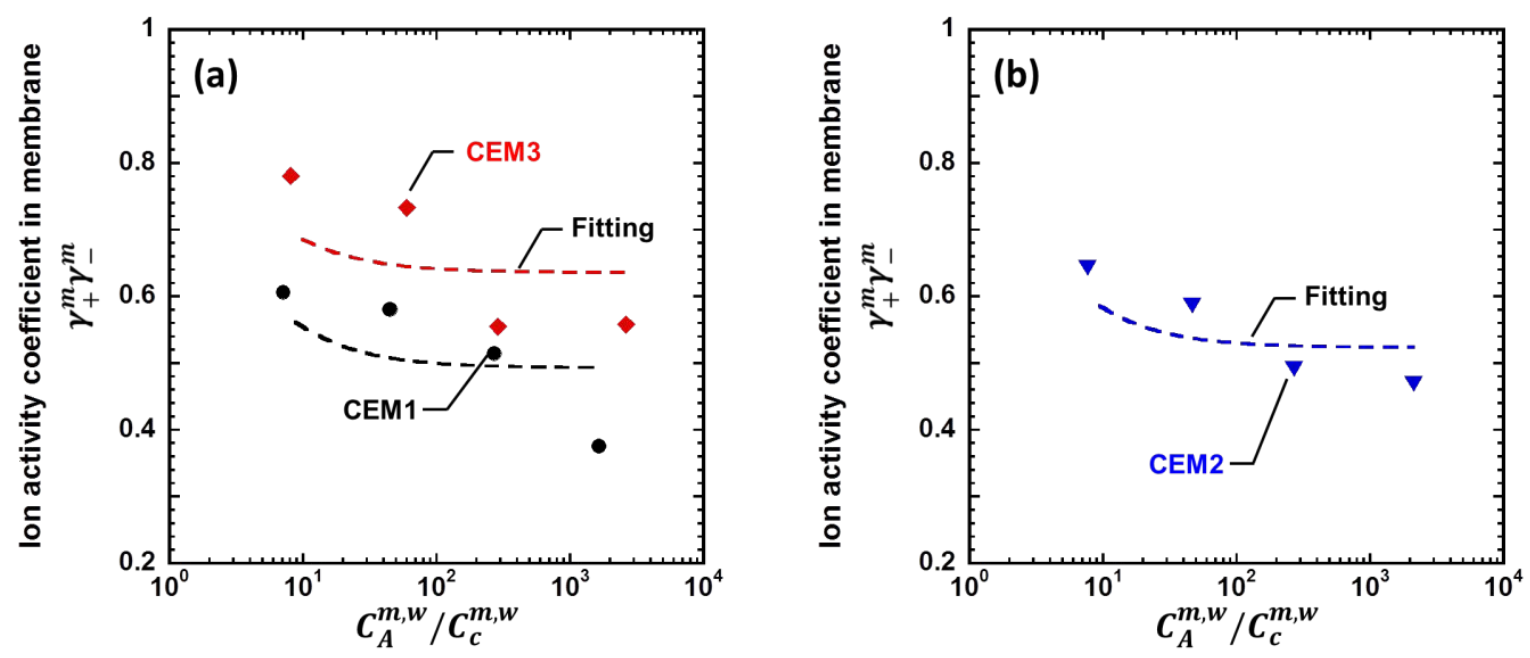

Figure S4. Ion activity coefficients in the membranes as a function of the ratio of fixed charge group concentration to the co-ion concentration in the membrane, $C_{A}^{m, w} / C_{s}^{m, w}$, for: (a) CEM1, CEM3, and (b) CEM2. The dashed curves were drawn using the Manning model for the case of $\xi$ $<1$. 
Table S5. Values for $C_{A}^{m, w} / C_{C}^{m, w}, \gamma_{+}^{m} \gamma_{-}^{m}$, $\xi$ as a function of external $\mathrm{NaCl}$ concentration, $C_{S}^{s}$.

\begin{tabular}{cccc}
\hline $\boldsymbol{C}_{\boldsymbol{s}}^{\boldsymbol{s}}[\mathbf{m o l} / \mathbf{L}]$ & $\boldsymbol{C}_{\boldsymbol{A}}^{\boldsymbol{m}, \boldsymbol{w}} / \boldsymbol{C}_{\boldsymbol{C}}^{\boldsymbol{m}, \boldsymbol{w}}$ & $\boldsymbol{\gamma}_{+}^{\boldsymbol{m}} \boldsymbol{\gamma}_{-}^{\boldsymbol{m}}$ & $\boldsymbol{\xi}$ \\
\hline & 9957.7 & 0.29 & 1.24 \\
$\mathbf{0 . 0 1}$ & 1660.3 & 0.37 & 0.98 \\
$\mathbf{0 . 0 3}$ & 271.6 & 0.51 & 0.67 \\
$\mathbf{0 . 1}$ & 45.1 & 0.58 & 0.57 \\
$\mathbf{0 . 3}$ & 7.1 & 0.61 & 0.64 \\
$\mathbf{1}$ & & & \\
& & 0.25 & 1.40 \\
$\mathbf{0 . 0 1}$ & 2116.1 & 0.47 & 0.76 \\
$\mathbf{0 . 0 3}$ & 269.7 & 0.49 & 0.72 \\
$\mathbf{0 . 1}$ & 46.8 & 0.59 & 0.56 \\
$\mathbf{0 . 3}$ & 7.7 & 0.64 & 0.56 \\
$\mathbf{1}$ & & & 1.66 \\
& & & 0.19 \\
$\mathbf{0 . 0 1}$ & 7083.4 & 0.56 & 0.59 \\
$\mathbf{0 . 0 3}$ & 2632.8 & 0.55 & 0.32 \\
$\mathbf{0 . 1}$ & 289.3 & 0.73 & 0.31 \\
\hline $\mathbf{1}$ & 80.1 & 0.78 & \\
\hline
\end{tabular}




\section{S8. Model fitting to SAXS scattering profiles for CEM2 and CEM3}
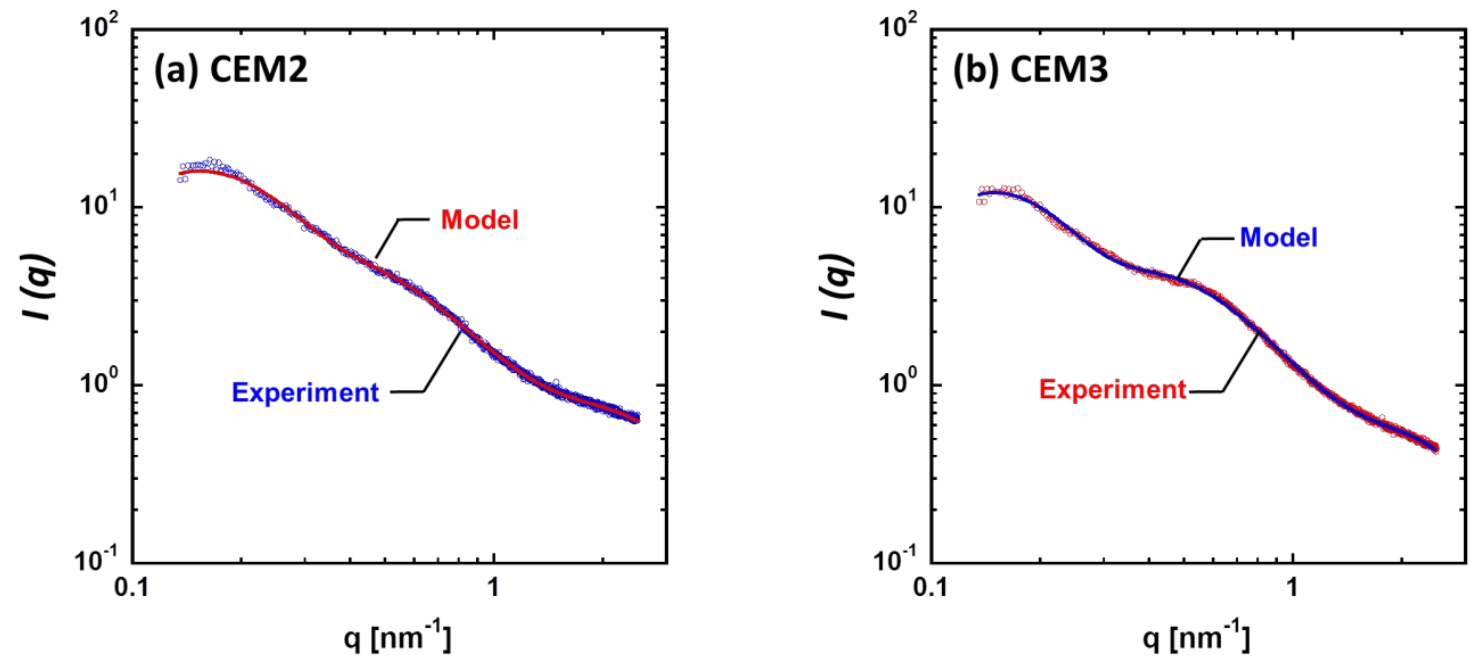

Figure S5. Model fit (eq 14 in the main text) to SAXS scattering profile for: (a) CEM2 and (b) CEM3. 


\section{S9. Membrane co-ion concentration predicted by Manning/Donnan model with various $\xi$ values}

To demonstrate the dependence of the predicted co-ion concentration on the Manning parameter, membrane co-ion concentrations for multiple Manning parameters are presented in Figure S6. The predicted co-ion concentrations using $\xi=1.24$ were approximately $25 \%$ greater on average than the predicted co-ion concentrations using $\xi=0.98$. These values for $\xi=0.98$ were approximately $45 \%$ greater on average that the predicted co-ion concentrations using $\xi=0.57$. Therefore, predicted membrane co-ion concentrations vary sensitively depending on Manning parameter.

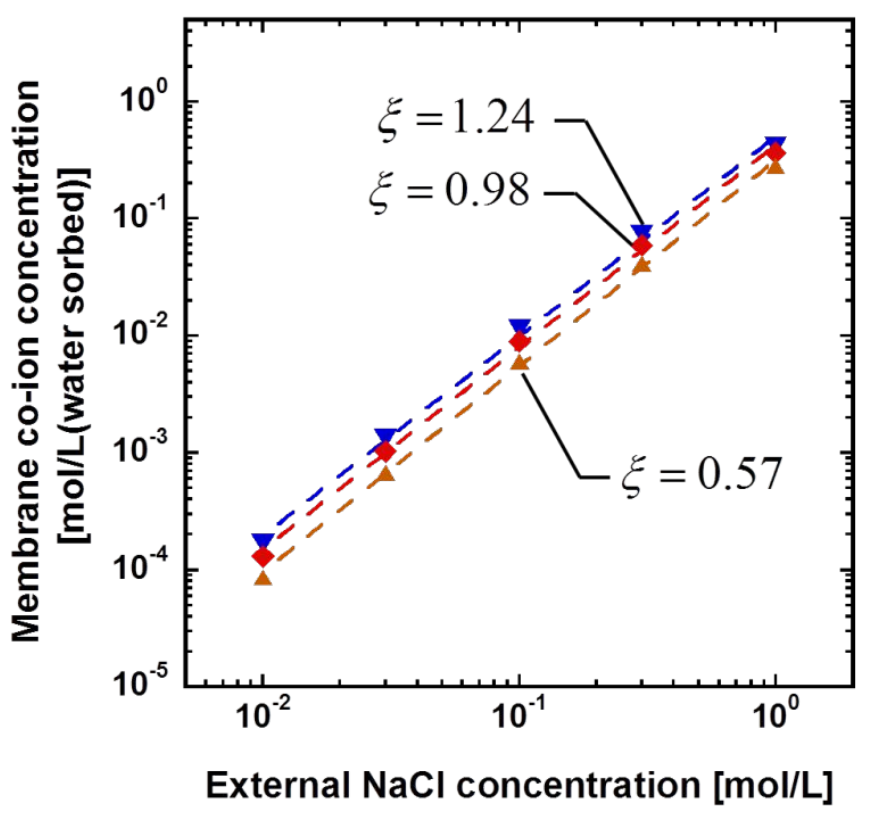

Figure S6. Membrane co-ion concentrations predicted by Manning/Donna model (eq 17 in the main text) as a function of external $\mathrm{NaCl}$ concentration for: $\xi=1.24, \xi=0.98$, and $\xi=0.57$. $C_{A}^{m, w}$ values for $\mathrm{CEM} 1$ at each external $\mathrm{NaCl}$ concentration were used for this calculation. 


\section{References}

(1) Xie, W.; Cook, J.; Park, H. B.; Freeman, B. D.; Lee, C. H.; McGrath, J. E. Fundamental salt and water transport properties in directly copolymerized disulfonated poly (arylene ether sulfone) random copolymers. Polymer 2011, 52 (9), 2032-2043.

(2) Kamcev, J.; Paul, D. R.; Freeman, B. D. Ion activity coefficients in ion exchange polymers: applicability of Manning's counterion condensation theory. Macromolecules 2015, 48 (21), 8011-8024.

(3) Kamcev, J.; Galizia, M.; Benedetti, F. M.; Jang, E. S.; Paul, D. R.; Freeman, B.; Manning, G. S. Partitioning of mobile ions between ion exchange polymers and aqueous salt solutions: importance of counter-ion condensation. Phys. Chem. Chem. Phys. 2016, 18 (8), 6021-6031. (4) Kamcev, J.; Paul, D. R.; Freeman, B. D. Effect of fixed charge group concentration on equilibrium ion sorption in ion exchange membranes. J. Mater. Chem. A 2017, 5 (9), 4638-4650. (5) Kwak, J. C. T. Mean activity coefficients for the simple electrolyte in aqueous mixtures of polyelectrolyte and simple electrolyte. System sodium polystyrenesulfonate-sodium chloride. J. Chem. Phys. 1973, 77 (23), 2790-2793.

(6) Paul, D. R., Polymer Blends. Academic Press: New York, 1978.

(7) Manning, G. S. Limiting laws and counterion condensation in polyelectrolyte solutions I. Colligative properties. J. Chem. Phys. 1969, 51 (3), 924-933. 